\title{
HEPATOPROTECTIVE ACTIVITY OF ETHANOLIC EXTRACT OF TERMINALIA CHEBULA FRUIT AGAINST ETHANOL INDUCED HEPATOTOXICITY IN RATS
}

\author{
BALAKRISHNA V*, LAKSHMI T \\ Department of Pharmacology, Guru Nanak Institutions Technical Campus, School of Pharmacy, Ibrahimpatnam, Hyderabad, Telangana, \\ India. Email: balakrishnavuyyala@gmail.com
}

Received: 27 May 2017, Revised and Accepted: 11 July 2017

\section{ABSTRACT}

Objective: The purpose of this study was to evaluate the effect of Terminalia chebula fruit extract on liver antioxidant enzymes in ethanol-induced hepatotoxicity in rats.

Method: Rats were divided into six different groups each having six. Group 1 served as a control, Group 2 received $40 \%$ ethanol ( 2 ml/100 g, oral), in sterile water, Groups 4, 5, and 6 served as extract treatment groups and received 50, 100, and $200 \mathrm{mg} / \mathrm{kg}$, orally, ethanolic fruit extract of T. chebula (TCE) and Group 3 served as standard group and received silymarin $25 \mathrm{mg} / \mathrm{kg}$ orally. All the treatment protocols followed 21 days, and after which rats were sacrificed, the liver was taken for antioxidant and histological studies, respectively.

Results: The ethanol-treated group rats (G2) showed variable decrease in antioxidant parameter (catalase, glutathione, and glutathione reductase) levels. Administration of ethanolic TCE significantly prevented ethanol-induced elevation in the levels of malondialdehyde lipid peroxidation and decreased antioxidant parameters in experimental groups of rats. The effect of extract was compared with a standard drug, silymarin. The changes in antioxidant parameters were supported by histological profile.

Conclusion: It is concluded that the ethanolic fruit TCE protects against ethanol-induced oxidative liver injury in rats.

Keywords: Terminalia chebula, Ethanol, Hepatotoxicity, Silymarin, Extract of Terminalia chebula.

(c) 2017 The Authors. Published by Innovare Academic Sciences Pvt Ltd. This is an open access article under the CC BY license (http://creativecommons. org/licenses/by/4. 0/) DOI: http://dx.doi.org/10.22159/ajpcr.2017.v10i11.20270

\section{INTRODUCTION}

Alcoholic liver disease (ALD) develops in patients consuming excessive amounts of alcohol. Alcohol dependency is not always a prerequirement for ALD development. In fact, some patients develop ALD and, in particular, cirrhosis without a history of dependence. Moreover, the severity of disease does not always correlate with the amount of alcohol intake, and environmental and genetic factors likely play a crucial role in ALD development. Although a dose-effect relationship between alcohol intake and alcohol-induced hepatic damage has been reported, there is no set amount of alcohol consumption that could surely predict the development of ALD [1]

In fact, the majority of long-term heavy drinkers develop fatty liver, but only $10-35 \%$ develop hepatitis and only $8-20 \%$ will progress to cirrhosis, and daily ethanol consumption exceeding 40-80 g/day for males and 20-40 g/day for females for 10-12 years will almost certainly lead to ALD. In a large survey conducted in Northern Europe, the relative risk of ALD significantly increased above a threshold of 7-13 drinks/week for women and 14-27 drinks/week in men [2].

Alcohol leads to increased liver oxidative stress through the generation of highly reactive oxygen species (ROS) and adducts. Alcohol dehydrogenase $(\mathrm{ADH})$ generates acetaldehyde, which is subsequently oxidized to acetate by aldehyde dehydrogenase (ALDH). Acetaldehyde can form hybrid-adducts with reactive residues (e.g. malondialdehyde [MDL] adduct) acting on proteins or small molecules (e.g. cysteines), mediating lipid peroxidation, and nucleic acid oxidation. Further oxidations in alcohol metabolism are accompanied by an excessive reduction of nicotinamide adenine dinucleotide (NAD), with a shift in the NADH/NAD ratio. Under normal circumstances, reduction of NAD (NAD/NADH) is finely regulated by the cell Krebs cycle. The shift caused by excessive alcohol consumption is thought to impair carbohydrate and lipid metabolism, finally causing impairment of gluconeogenesis and diversion of metabolism to ketogenesis and fatty acid synthesis. The increased amount of reducing equivalents, such as NADH, leads to their shuttering into mitochondria, which induces the electron transport chain components to assume a reduced state. This facilitates the transfer of an electron to molecular oxygen to generate reactive species as superoxide anion. Mitochondrial ROS generation can also derive from the alterations produced in mitochondrial complexes I and III, which have been discussed above. In fact, such alteration can also promote superoxide anion generation within the mitochondria [3].

Thus, mitochondria represent a main site where huge amount of ROS are generated, leading, in turn, to cell damage and necrosis. Finally, the NADH-induced inhibition of mitochondrial b-oxidation leads to accumulation of intracellular lipids, thus promoting steatosis. Excessive alcohol consumption is also associated with the enzymatic induction of CYP2E1 pathway of alcohol metabolism. The recruitment of this pathway may indirectly contribute to ALD development by excess production of superoxide radicals through the interaction of CYP2E1 with cytochrome reductase, which leads to electron leaks in the respiratory chain and ROS production. The species produced in this cascade can interact with iron (Fenton reaction) generating even more potent hydroxyl, ferryl, and perferryl radicals which perpetuate liver damage $[4,5]$.

Terminalia chebula fruits contain wide range of tannins, triterpenoids, glycosides, anthraquinones, and flavonoids. Due to its antioxidant activity it protects the body's cells and DNA from free radical damage [6]. The present investigation was undertaken to study the effect of T. chebula fruit extract on liver antioxidant enzymes in ethanolinduced hepatotoxicity in rats.

\section{METHODS}

\section{Collection and authentication of plant}

T. chebula fruits were freshly collected from the medicinal plants farm, Mulugu, Warangal surroundings Telangana. The plant material was taxonomically identified and authenticated by Mr. P. V. Prasanna, Scientist 'E'/Officer In-charge, BSI, Govt. of India and the voucher specimen No.BSI/DRC/2014-2015/Tech/746. 
Chemicals

MDL lipid peroxidation, catalase (CAT), glutathione (GSH), and glutathione reductase (GR) were assayed using kits. All the drugs, chemicals, and reagents used for biochemical estimation were purchased from Sigma-Aldrich, USA.

\section{Animals}

Male Wistar albino rats, weighing about 150-200 g, were obtained from the National Institute of Nutrition, Hyderabad and used in the experiments. The protocol was approved by the Institutional Animal Ethical Committee (Reg no: MRCP/CPCSEA/IAEC/2013-14/ MPCOL/11). Animals were kept in the animal house at an ambient temperature of $25^{\circ} \mathrm{C}$ and $45-55 \%$ relative humidity, with $12 \mathrm{hrs}$ each of dark and light cycles. Animals were fed pellet diet and water ad-libitum.

Acute toxicity study was conducted for both the extracts by stair case method [7]. The $\mathrm{LD}_{50}$ of ethanol and aqueous leaf extracts were found to be $500 \mathrm{mg} / \mathrm{kg}$ per orally (p.o.) and $1000 \mathrm{mg} / \mathrm{kg}$ p.o. and $2000 \mathrm{mg} / \mathrm{kg}$, one-tenth of this was selected as maximum dose for the evaluation of antihepatotoxic activity [8], i.e., $50 \mathrm{mg} / \mathrm{kg}$ p.o., $100 \mathrm{mg} / \mathrm{kg}$ p.o., and $200 \mathrm{mg} / \mathrm{kg}$

\section{Induction of experimental hepatotoxicity}

Rats were treated with $40 \%$ ethanol ( $2 \mathrm{ml} / 100 \mathrm{~g}$, orally) for 21 days to study the effect of ethanolic which was used as a standard drug in this study.

\section{Treatment protocol}

Thirty-six Wistar Albino male rats of weight 150-250 g were selected for this study. Animals were divided into six groups of six animals each.

Group 1: Control group (distilled water) for 21 days.

Group 2: Inducer (ethanol $2 \mathrm{ml} / 100 \mathrm{~g}$ body weight, p.o.) for 21 days.

Group 3: T. chebula fruit extract $50 \mathrm{mg} / \mathrm{kg}$ body weight p.o. + ethanol $2 \mathrm{ml} / 100 \mathrm{~g}$ body weight, p.o. for 21 days.

Group 4: T. chebula fruit extract $100 \mathrm{mg} / \mathrm{kg}$ body weight p.o. + ethanol $2 \mathrm{ml} / 100 \mathrm{~g}$ body weight, p.o. for 21 days.

Group 5: T. chebula fruit extract $200 \mathrm{mg} / \mathrm{kg}$ body weight p.o. + ethanol $2 \mathrm{ml} / 100 \mathrm{~g}$ body weight, p.o. for 21 days.

Group 6: Silymarin $25 \mathrm{mg} / \mathrm{kg}$ body weight p.o. + ethanol $2 \mathrm{ml} / 100 \mathrm{~g}$ body weight, p.o. for 21 days.

\section{Biochemical estimation}

At the end of the study, animals were sacrificed by cervical dislocation with ether anesthesia; the liver tissue was separated and washed with phosphate-buffered saline $(0.05 \mathrm{M}, \mathrm{pH}: 7.4)$. The liver tissue was taken and minced into small pieces and homogenized in ice-cold phosphate- buffered saline $(0.05 \mathrm{M}, \mathrm{pH}: 7.4)$ using tissue homogenizer to obtain $1: 9(\mathrm{w} / \mathrm{v})(10 \%)$ whole homogenate. A part of the liver homogenate was taken and mixed with equal volume of $10 \%$ trichloroacetic acid (TCA) for the estimation of MDL. Homogenate was centrifuged using Remi cool centrifuge at $8000 \mathrm{rpm}$ for 30 minutes. The supernatant was separated and used for estimation of MDL [9] and antioxidant levels of different enzymes, i.e., CAT [10], GR, and reduced GSH [11] in the liver tissue.

\section{Histopathological studies}

At the end of the experiment (day 21), all rats were anesthetized by ether, and the liver was excised out and fixed in formalin (10\%). Five micron thick sections were prepared using microtome, and these sections were stained with hematoxylin and eosin. For histological alterations, these slides were observed under light microscope.

\section{Statistical analysis}

The results are expressed as mean \pm standard error of the mean. The evaluation of the data was done using one-way ANOVA followed by Dunnett's multiple comparisons tests. $\mathrm{p}<0.01$ was considered statistically significant.

\section{RESULTS}

\section{Histopathology}

Hepatocytes are normal in cell morphology with moderate eosinophilic cytoplasm contains fine chromatin. Hepatic vein, central vein, and portal triads are in normal in position (Fig. 1a).

There is ballooning degeneration of hepatocytes with patchy and perivascular inflammatory component comprising lymphomononuclear cell aggregates. There is mild central vein dilation, massive fatty changes and broad infiltration of the lymphocytes and the loss of cellular boundaries seen in toxicant ethanolic group [12] (Fig. 1b).

The liver section show still persisting of degenerated hepatocytes with sparse inflammatory component. Bile duct, central vein, and portal triad are normal in TCE $(50 \mathrm{mg} / \mathrm{kg})$ (Fig. 1c).

The liver section show disappearance of fibrous septae and hepatocytes appeared to be normal. Very less mononuclear inflammatory infiltration is found in TCE $(100 \mathrm{mg} / \mathrm{kg})$ (Fig. 1d).

The liver section shows still persisting of less degenerated hepatocytes with sparse inflammatory component. Bile duct, central vein, and portal triad are normal in TCE (200 mg/kg) [13] (Fig. 1e).

The liver section shows hepatocytes are normal in cell pattern and arrangement. No inflammatory component, no thromobosis, portal triad, central vein, and bile duct are more or less normal in standard silymarin $(25 \mathrm{mg} / \mathrm{kg})$ (Fig. 1f).
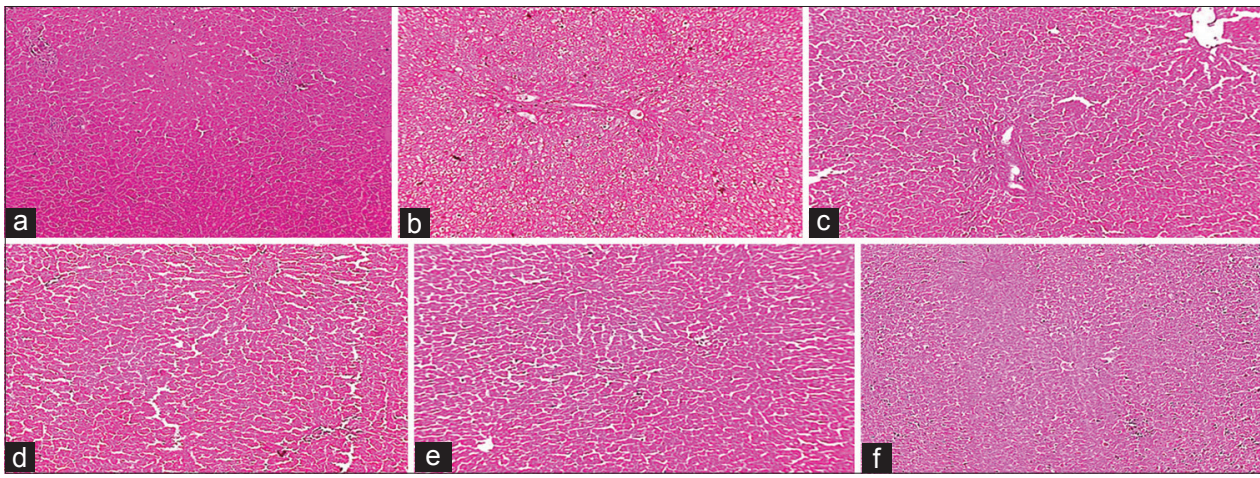

Fig. 1: (a) Normal control. (b) Inducer ethanol. (c) Extract of Terminalia chebula (TCE) (50 mg/kg). (d) TCE (100 mg/kg). (e) TCE (200 mg/kg). (f) Standard silymarin $(25 \mathrm{mg} / \mathrm{kg})$ 
Table 1: Effect of TCE on antioxidant parameters (MDL, GSH, CAT, and GR) ethanol-induced hepatotoxicity in rats

\begin{tabular}{|c|c|c|c|c|c|}
\hline Groups & Treatment & MDL (nmol/g) & $\mathrm{GSH}(\mu \mathrm{mol} / \mathrm{min} / \mathrm{mg})$ & CAT $(\mu \mathrm{mol} / \mathrm{min} / \mathrm{mg})$ & GR $(\mathrm{U} / \mathrm{ml})$ \\
\hline I & Normal control & $53.33 \pm 2.108$ & $21.58 \pm 0.510$ & $20.1 \pm 0.03$ & $34.6 \pm 0.22$ \\
\hline II & Inducer (ethanol 40\%) & $107.24 \pm 8.83^{* * *}$ & $13.88 \pm 0.38^{* * *}$ & $10.3 \pm 0.045^{* * *}$ & $20.5 \pm 0.4^{* * *}$ \\
\hline III & TCE (50 mg/kg)+ethanol (40\%) & $92.33 \pm 2.2^{*}$ & $16.14 \pm 0.17^{*}$ & $12.2 \pm 0.010^{* *}$ & $25.6 \pm 0.5^{*}$ \\
\hline IV & TCE $(100 \mathrm{mg} / \mathrm{kg})+$ ethanol $(40 \%)$ & $73.67 \pm 1.89 * *$ & $19.24 \pm 0.220^{* *}$ & $15.4 \pm 0.044^{*}$ & $29.6 \pm 0.4^{* *}$ \\
\hline V & TCE (200 mg/kg)+ethanol (40\%) & $56.67 \pm 2.61^{* *}$ & $21.42 \pm 0.344^{* *}$ & $18.5 \pm 0.021^{* *}$ & $31.6 \pm 0.3^{* *}$ \\
\hline VI & Silymarin (25 mg/kg)+ethanol 40\% & $49.0 \pm 2.27^{* *}$ & $26.12 \pm 0.155^{* *}$ & $17.8 \pm 0.028^{* *}$ & $33.5 \pm 0.3^{* *}$ \\
\hline
\end{tabular}

Values are expressed as mean \pm SEM, $n=6$. The values were determined using one-way ANOVA followed by Dunnett's test. ${ }^{* * *}<<0.001$ as compared with normal control. ${ }^{*} \mathrm{p}<0.05,{ }^{* *} \mathrm{p}<0.01$ as compared with inducer ethanol. MDL: Malondialdehyde, GSH: Glutathione, CAT: Catalase, GR: Glutathione reductase, TCE: Extract of Terminalia chebula

\section{DISCUSSION}

Alcohol is one of the most important and commonly used hepatotoxic agents in the experimental study of liver-related disorders. The hepatotoxic effects of alcohol are largely due to its active metabolite trichloromethyl radical. This activated radical bind covalently to the macromolecules and induces peroxidative degradation of membrane lipids of endoplasmic reticulum rich in polyunsaturated fatty acids. This process leads to excessive formation and accumulation of lipids in tissues such as the liver. Lipids from peripheral adipose tissue are translocated to the liver for accumulation $[14,15]$.

The oxidation of ethanol to water and carbon dioxide is mediated by three major hepatic enzyme systems: ADH in cytoplasm, microsomal ethanol oxidizing system in smooth endoplasmic reticulum of mitochondria (predominantly CYP2E1), and CAT in peroxisomal membrane. All these biochemical pathways produce acetaldehyde as their toxic by-products.

Free radicals (ROS and reactive nitrogen species) are generated from our body by various endogenous systems, exposure to different physiochemical conditions, or pathological states. A balance between free radicals and antioxidants is necessary for proper physiological function. If the free radicals overwhelm the body's ability to regulate them, a condition known as oxidative stress ensues. Free radicals thus adversely alter lipids, proteins, and DNA and trigger a number of disorders in human being.

Exposure to free radicals from a variety of sources has led organisms to develop a series of defense mechanisms. Defense mechanisms against free radical-induced oxidative stress involve:

i. Preventative mechanisms,

ii. Repair mechanisms,

iii. Physical defenses,

iv. Antioxidant defenses.

Enzymatic antioxidant defenses include MDL lipid peroxidation, glutathione peroxidase (GPx), CAT. Non-enzymatic antioxidants are represented by ascorbic acid (Vitamin C), tocopherol (Vitamin E), GSH, carotenoids, flavonoids, and other antioxidants.

GSH is a cofactor of several detoxifying enzymes against oxidative stress, e.g., GPx, glutathione transferase, and others. GSH scavenges hydroxyl radical and singlet oxygen directly, detoxifying hydrogen peroxide, and lipid peroxides by the catalytic action of glutathione peroxidase. GSH is able to regenerate the most important antioxidants, Vitamins $\mathrm{C}$ and $\mathrm{E}$, back to their active forms; GSH can reduce the tocopherol radical of Vitamin $\mathrm{E}$ directly, or indirectly, through reduction of semidehydroascorbate to ascorbate. The capacity of GSH to regenerate the most important antioxidants is linked with the redox state of the glutathione disulfide (GSSG)-2GSH [14].

CAT is an enzymatic antioxidant widely distributed in all animal tissue, and the highest activity is found in the red cells and in the liver. CAT decomposes hydrogen peroxide and protects the tissue from highly reactive hydroxyl radicals (Table 1) [15]. Ethanol exposed rats exhibited decrease in CAT level when compared to normal group. Treatment with various doses of $T$. chebula fruit extract significantly increased CAT levels when compared to ethanolic group.

GR, also known as GSR or GR, is an enzyme that reduces GSSG to the sulfhydryl form GSH, which is an important cellular antioxidant. The levels of GR levels were monitored by Valko et al. [16]. Ethanol exposed rats exhibited significantly decrease in GR levels when compared to normal group. Treatment with various doses of T. chebula fruit extract significantly increased GR levels when compared to the ethanolic group.

Alcohol leads to increased liver oxidative stress through the generation of highly ROS and adducts. ADH generates acetaldehyde, which is subsequently oxidized to acetate by ALDH. Acetaldehyde can form hybrid adducts with reactive residues (e.g. MDL adduct) acting on proteins or small molecules (e.g. cysteines), mediating lipid peroxidation, and nucleic acid oxidation. Further oxidations in alcohol metabolism are accompanied by an excessive reduction of NAD, with a shift in the NADH/NAD ratio. Under normal circumstances, reduction of NAD (NAD fi NADH) is finely regulated by the cell Krebs cycle $[17,18]$.

The protective action of antioxidants is usually due to the inhibition of free radical chain reaction and the resultant prevention of peroxidative deterioration of structural lipids in membranous organelles $[19,20]$. The enzymatic and non-enzymatic such as MDL lipid peroxidation, CAT, and reduced GSH play an important role in alleviating tissue damage due to the formation of free radicals $[21,22]$.

On morphological examination in low dose, T. chebula showed partial inflammation in hepatic cells while in high dose $(200 \mathrm{mg} / \mathrm{kg})$, extract of T. chebula (TCE) fruit showed a highly recovery compared to normal.

The hepatoprotective role of ethanolic TCE fruit might be due to its antioxidant potential mechanism suggesting that the extract of plant may be useful to prevent the ethanol induced hepatotoxicity [23]. More research is required in this viewpoint to develop a good hepatoprotective drug from fruits of $T$. chebula. The purification of extract and identification of the active principle may yield active hepatoprotective ingredients.

\section{CONCLUSION}

The present study opens many new areas of research work. This work can be continued in the future to study and confirm liver protective activity in different experimental models and also to isolate, identify, characterize, and standardize the active principle(s) that are responsible for this activity. The antioxidant activity of TCA might be responsible for its importance in traditional medicine for the treatment of various disorders.

\section{REFERENCES}

1. Mandayam S, Jamal MM, Morgan TR. Epidemiology of alcoholic liver disease. Semin Liver Dis 2004;24(3):217-32.

2. Roizen R, Kerr WC, Fillmore KM. Cirrhosis mortality and per capita consumption of distilled spirits, United States, 1949-1994: trend 
analysis. West J Med 1999;171(2):83-7.

3. Suguna L, Singh S, Sivakumar P, Sampath P, Chandrakasan G. Influence of Terminalia chebula on dermal wound healing in rats. Phytother Res 2002;16(3):227-31.

4. Wu D, Cederbaum AI. Alcohol, oxidative stress, and free radical damage. Alcohol Res Health 2003;27(4):277-84.

5. Thurman RG. Mechanism of hepatic toxicity: II. Alcoholic liver injury involves activation of Kupffer cells by endotoxin. Am J Physiol 1998;275:G605-11.

6. Hazra B, Sarkar R, Biswas S, Mandal N. Comparative study of the antioxidant and reactive oxygen species scavenging properties in the extracts of the fruits of Terminalia chebula, Terminalia bellirica and Emblica officinalis. BMC Complement Altern Med 2010;10:20.

7. Kokate CK, Purohith AP, Gokhale SB. Pharmacognosy. Pune: Nirali Prakashan; 1990.

8. Ghosh MN. Fundamentals of Experimental Pharmacology. Kolkata: Scientific Book Agency; 1984.

9. Bergmeyer HU, Hørder M, Rej R. International Federation of Clinical Chemistry (IFCC) Scientific Committee, Analytical Section: Approved recommendation (1985) on IFCC methods for the measurement of catalytic concentration of enzymes. Part 3. IFCC method for alanine aminotransferase (L-alanine: 2-oxoglutarate aminotransferase, EC 2.6.1.2). J Clin Chem Clin Biochem 1986;24(7):481-95.

10. Georg LE. Tissue sulfhydryl group. Arch Biochem Biophy 1959;82(1):70-7.

11. Saravanan N, Nalini N. Antioxidant effect of Hemidesmus indicus on ethanol-induced hepatotoxicity in rats. J Med Food 2007;10(4):675-82.

12. Jain A, Soni M, Deb L, Jain A, Rout SP, Gupta VB, et al. Antioxidant and hepatoprotective activity of ethanolic and aqueous extracts of Momordica dioica Roxb. Leaves. J Ethnopharmacol 2008;115(1):61-6.

13. Evan PS, Mahaboobkhan R, Mahima V, Dhanalakshmi N, Meenakshi R,
Poornima $\mathrm{P}$, et al. Hepatoprotective and antioxidant potential of Withania somnifera against paracetamol-induced liver damage in rats. Int J Pharm Pharm Sci 2013;10(2):648-51.

14. Monzoni A, Masutti F, Saccoccio G, Bellentani S, Tiribelli C, Giacca M. Genetic determinants of ethanol-induced liver damage. Mol Med 2001;7(4):255-62.

15. Rajalingam D, Varadharajan R, Palani S. Evaluation of hepatoprotective and antioxidant effect of Combretum albidum g. Don against ccl4 induced hepatotoxicity in rats. Int J Pharm Pharm Sci 2016;8(9):218-23.

16. Valko M, Leibfritz D, Moncol J. Free radical and antioxidants in normal physiological functions and human disease. J Biocell 2006;7(1):45-78.

17. Chance B, Greenstein DS, Roughton FJ. The mechanism of catalase action. I. Steady-state analysis. Arch Biochem Biophys 1952;37(2):301-21.

18. Venditti P, Balestrieri M, Di Meo S, De Leo T. Effect of thyroid state on lipid peroxidation, antioxidant defences, and susceptibility to oxidative stress in rat tissues. J Endocrinol 1997;155(1):151-7.

19. Stewart S, Jones D, Day CP. Alcoholic liver disease: New insights into mechanisms and preventative strategies. Trends Mol Med 2001;7(9):408-13

20. Reinke LA, Lai EK, DuBose CM, McCay PB. Reactive free radical generation in vivo in heart and liver of ethanol-fed rats: Correlation with radical formation in vitro. Proc Natl Acad Sci U S A 1987;84(24):9223-7.

21. Finkel T, Holbrook NJ. Oxidants, oxidative stress and the biology of ageing. Nature 2000;408(6809):239-47.

22. Priyanka S, Anupam DT, Manabendra DC. Antioxidant potential profile of Pajanelia longifolia (Willd.) k. Schuman; Potential new sources of natural antioxidant. Asian J Pharm Clin Res 2017;10(6):184-8.

23. Sarita MK, Abhijit B. Standardization of model of induction of hepatotoxicity with anti tuberculosis drugs in wistar albino rats. Asian J Pharm Clin Res 2017;10(6):150-3. 